\title{
Brain Amyloid and Inflammation Imaging: A Convergence of Concepts
}

\author{
J. James Frost
}

Published online: 10 July 2013

(c) Springer Science+Business Media New York 2013

\begin{abstract}
Molecular imaging in the brain and other organs continues to advance understanding of disease mechanisms, new targets for treatment, and new diagnostic biomarkers. Brain amyloid and inflammation imaging are two areas of intense investigation. Progress in amyloid imaging has resulted in one approved PET tracer and several others in clinical development. Current efforts focus on early treatment with amyloid reducing drugs in patients who are amyloid scan positive prior to the onset of fully developed dementia. Inflammation imaging is primarily directed toward translocator protein imaging in multiple sclerosis and other inflammatory brain disorders. Alzheimer's disease (AD), having an inflammatory component, is receiving increased attention from the standpoint of the genetics of inflammation and the use of inflammation imaging agents. A convergence of interest in the role of inflammation and amyloid deposition in $\mathrm{AD}$ may elucidate new disease mechanisms and advance therapeutic and diagnostic approaches.
\end{abstract}

Keywords Positron emission tomography - PET · Imaging · Amyloid · Alzheimer's disease · Inflammation · Multiple sclerosis

\section{Introduction}

Molecular imaging ofthe brain originated in the early 1980s when the first methods were developed to image

J. J. Frost $(\bowtie)$

BioMolecular Imaging, LLC and John Hopkins University

School of Medicine, 3801 Canterbury Road, Baltimore,

MD 21218, USA

e-mail: JamesFrost@BioMolecularImaging.com neuroreceptors, including the dopamine and opiate receptors, by PET imaging. Subsequently, radiolabeled tracers for a myriad of other neuroreceptors, their receptor subtypes, neurotransmitter transporters, and enzymes were developed. In the twenty-first century molecular imaging of brain targets continues to be very active, in part due to the many prevalent brain disorders whose mechanistic understanding is incomplete and for which therapies are inadequate. Among these, dementia of the Alzheimer type remains a major treatment challenge. Fortunately, brain imaging technologies are surpassing the state of therapy development and may provide a new avenue for understanding disease mechanisms and advancing new treatment approaches. The major advance is in brain amyloid imaging, focusing, as do the current treatment efforts, on the amyloid hypothesis of Alzheimer's disease (AD).

In parallel, inflammation imaging in the brain and other organs is advancing at a rapid rate. This relatively recent effort is the result of the increasing recognition of the role of inflammatory processes in many disease processes, including in neurologic, psychiatric, cardiovascular, metabolic, and musculoskeletal disorders, coupled with the feasibility of inflammatory target molecular imaging. The translocator protein (TSPO) target has received the greatest attention and many new imaging probes have been developed, including one for commercialization. Although the current focus of TSPO imaging is in the brain, this target has application in other organ systems and diseases as well. Inflammation is also thought to play a role in $\mathrm{AD}$, resulting from amyloid deposition or alternatively, as a precursor or possible cause of amyloid synthesis. Recent genetic studies increasingly support a role for inflammation and suggest new approaches to imaging and treatment, possibly overcoming the limitations of current anti-amyloid treatments. Alternatively, early detection of brain amyloid may permit 
early treatment with anti-amyloid drugs and result in greater efficacy compared to that in established AD. This mini-review will provide an update to the stories that are currently playing out in amyloid and inflammation imaging and point to a direction where the concepts may converge to advance diagnosis and treatment in prevalent diseases with inadequate treatments.

\section{Amyloid Imaging}

After a considerable period of development and scientific investigation, brain amyloid imaging has reached the stage of commercialization and progressive implementation into clinical practice. Four ${ }^{18}$ F-labeled amyloid imaging tracers are undergoing clinical development: florbetapir, florbetaben, flutemetamol, and NAV4694 (formerly AZD4694) [1$4 \bullet, 5]$. These agents are all labeled with ${ }^{18} \mathrm{~F}\left(t_{1 / 2}=2 \mathrm{~h}\right)$ to permit delivery to imaging sites from a commercial pharmacy. Florbetapir has been approved by the Food and Drug Administration for use in humans for detecting the presence of amyloid plaques. All of these agents are able to demonstrate the presence of amyloid in patients with established AD. Some individuals, however, may have amyloid deposits by imaging and show no development of $\mathrm{AD}$ for long periods of time. In a recent study, elderly dementia-free subjects aged 82-95 were imaged with ${ }^{11} \mathrm{C}$ PIB. Amyloid positivity was related to a faster reduction in executive functioning in the 7-9 year period prior to the PET scan, indicating a possible functional effect of brain amyloid in otherwise healthy very old individuals [6]. At present, however, the current challenge is still to improve knowledge of the false-positive and -negative rates for diagnosis of $\mathrm{AD}$ in patients with early signs of dementia, such as in mild cognitive impairment (MCI) or in subjects with a genetic risk of $\mathrm{AD}$. Commercial image processing and analytics software applications implemented on dedicated workstations and new guidelines for clinician image reads are also being developed. In parallel, studies are ongoing to compare the performance characteristics of the various amyloid imaging probes (Fig. 1) [4•].

The lack of an effective disease-modifying treatment for $\mathrm{AD}$ has continued to limit the utility of imaging diagnostics, but benefits other than treatment-related are claimed, including an improved ability to plan ones life and affairs. The failure of brain amyloid-reducing treatments to improve cognition has cast uncertainty on the amyloid hypothesis of $\mathrm{AD}$, but many believe that detection of early amyloid brain deposition using molecular imaging could lead to early therapy implementation and improved outcome. It is possible that administration of amyloid-reducing drug therapy in early cognitive impairment with a low amyloid burden demonstrated by imaging could result in the reversal of cognitive decline or decreasing the rate of decline. Studies are currently underway to examine this hypothesis.

Currently, the Alzheimer's Association and the Society for Nuclear Medicine and Molecular Imaging have published a set of guidelines for the use of amyloid imaging based on the information available from existing clinical trials. This is the first guidance for any imaging technology applied to $\mathrm{AD}\left[\mathrm{7}^{\circ}\right]$. The guidance concludes that the greatest benefit will be for (1) patients with persistent or progressive unexplained MCI; (2) patients satisfying core clinical criteria for possible AD because of unclear clinical presentation, either an atypical clinical course or an etiologically mixed presentation; and (3) patients with progressive dementia at an atypically early age of onset (usually defined as 65 years or less in age). Correspondingly, a number of scenarios are summarized where imaging would not be justified, including for determination of the severity of dementia and in asymptomatic individuals.

A necessary condition for widespread use of amyloid imaging in clinical practice is insurance reimbursement, which would be initially on the order of $\$ 3,000$ for the technical and professional costs. A recent meeting of the Medicare Evidence Development and Coverage Advisory Committee (MEDCAC) panel was convened on 30 January 2013 to offer guidance to the Centers for Medicare and Medicaid Services in consideration of amyloid imaging reimbursement [8]. The confidence level on the part of the committee was low to intermediate that the test had sufficient power to identify $\mathrm{AD}$ in the group of patients with early onset cognitive impairment. At issue was the observation that a significant fraction, perhaps up to $30 \%$ of individuals may have elevated amyloid by imaging and never develop AD. Ongoing studies will continue to define the false positive rate for the imaging test, but it is likely that the predictive value of a negative test will be greater than the predictive value of a positive test. Accordingly, subjects with MCI and a negative amyloid imaging study will have a low likelihood of developing $\mathrm{AD}$, but the precise false-negative rate needs to be elucidated through ongoing clinical trials. There may be value to patients and their families to know in advance the likelihood of developing $\mathrm{AD}$ even in the absence of a disease-modifying treatment [7•]. The MEDCAC, however, pointed out the lack of any study documenting such value. Therefore, it is likely that investigators will begin to examine this question while new therapeutic drugs are developed.

\section{Inflammation Imaging}

Inflammation is believed to play a causal role in many disorders, including in neurologic, psychiatric, cardiovascular, 


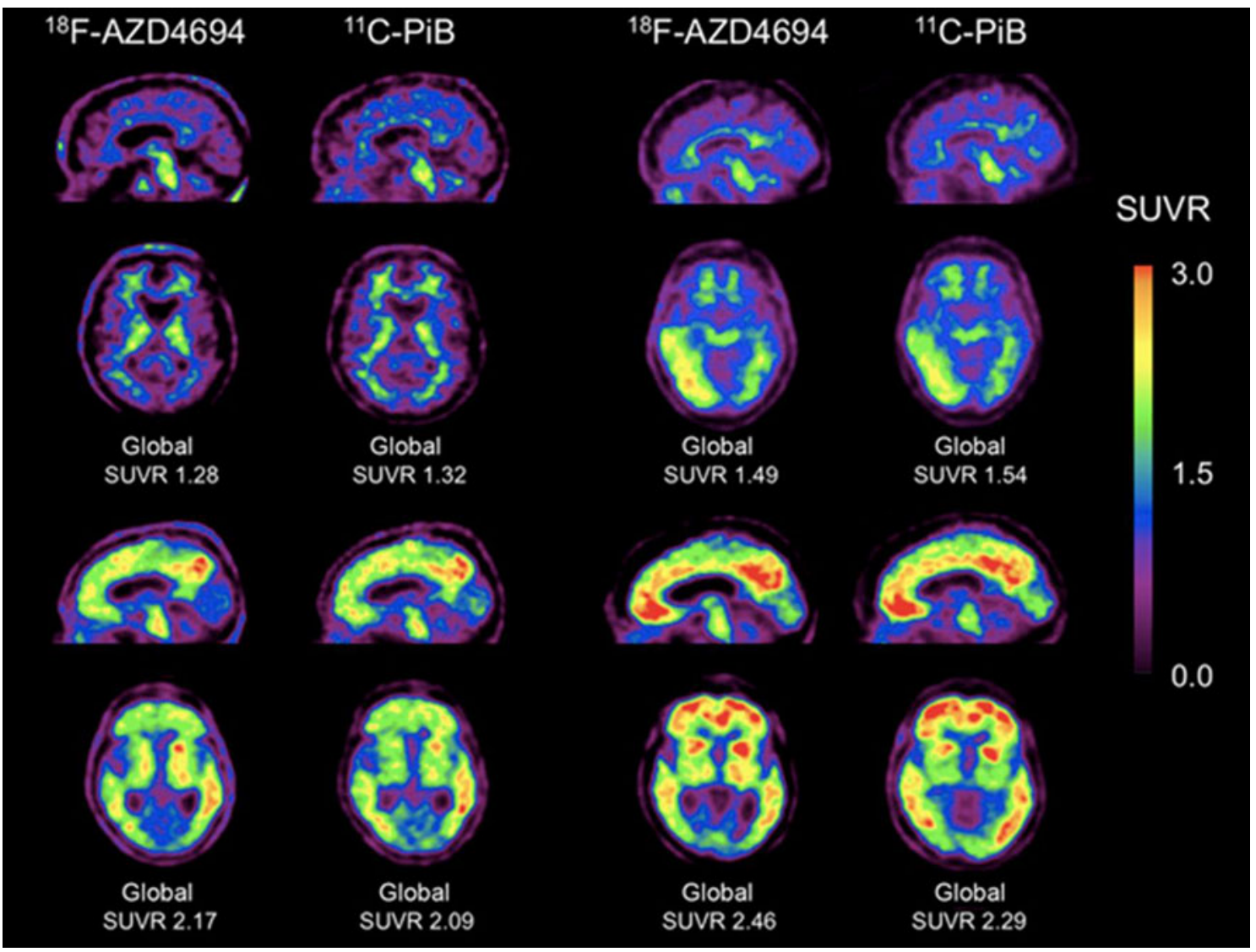

Fig. $1{ }^{18} \mathrm{~F}$-AZD4694 and ${ }^{11} \mathrm{C}$-PIB PET imaging in four subjects representative of range of tracer binding. The top two rows show binding in two healthy controls, demonstrating the normal white matter binding and low cortical uptake. The bottom two rows show imaging from two patients with clinical $\mathrm{AD}$, showing the enhanced

metabolic, musculoskeletal, and respiratory diseases. Among these, the importance of brain inflammation in multiple sclerosis, depression, Huntington's disease, Parkinson's disease and ADs has been recognized. Accordingly, the recent progress in developing molecular probes for inflammation imaging has predominantly occurred for the brain.

The target of greatest interest for imaging is the TSPO, which exists on the outer mitochondrial membrane and is responsible for the transport of cholesterol and the eventual synthesis of pregnenolone. It is highly expressed in macrophages, activated microglia and reactive astrocytes [9]. The original TSPO imaging agent was ${ }^{11} \mathrm{C}-\mathrm{PK} 11195$, developed as an imaging agent for the peripheral benzodiazepine receptor, which is identical to the TSPO. In an effort to optimize TSPO agents for brain imaging and reduced white matter binding ${ }^{11} \mathrm{C}-\mathrm{PBR} 28$ and follow-on tracers were developed [10]. Thereafter, a number of ${ }^{18} \mathrm{~F}$-labeled probes have been developed, including ${ }^{18} \mathrm{~F}$-PBR $111,{ }^{18} \mathrm{~F}$-DPA714, ${ }^{18}$ F-FEDAA1106. ${ }^{18}$ F-GE180 is a commercial TSPO imaging agent currently undergoing first-in-human trials for eventual use in multiple sclerosis and other inflammatory uptake in the cortical gray matter areas. For these two tracers the binding patterns are nearly identical in appearance and dynamic range using the standardized uptake value ratios (SUVR) (from [4•], with permission)

disorders [11]. All of these probes recognize the high- and low-affinity states of TSPO, which vary as a function of individual genetics. At present, therefore, imaging data interpretation must rely on independent knowledge of TSPO gene status of each subject [12].

Multiple sclerosis is a disease characterized by microglial activation and neural degeneration. It was one of the first disorders to be evaluated using TSPO ligands and continues to be an area of active investigation due to the limitations of current imaging methods to monitor disease activity and treatment response (Fig. 2). A number of studies have demonstrated significant correlations between TSPO binding and clinical symptoms, including disease severity and duration [13]. Another study contradicts these results, but tracer, patient population, and genetic differences may account for the different results [14]. Studies are also evaluating the overlap of focal TSPO binding and lesion enhancement on MR [15]. An experimental model in baboons has recently demonstrated that $E$. coli lipopolysaccharide-induced inflammation results in elevated TSPO binding, permitting more detailed experimental analysis of 
Fig. 2 Gadolinium contrastenhancing lesions show focal increase in [11C]PBR28 binding. a Fluid-attenuated inversion recovery (FLAIR) and post-contrast T1-weighted (post-contrast T1) MRI, and [11C]PBR28 PET from a subject with MS (MS3) showing increased [11C]PBR28 binding in the areas corresponding to gadolinium-enhancing lesion (arrows). b [11C]PBR28 binding in gadolinium contrastenhancing lesions (CELs) compared to contralateral normal-appearing white matter (NAWM). Error bars indicate \pm SD. c MRI coregistered baseline-to-follow-up [11C]PBR28 VT difference map (left) and post-contrast T1weighted MRI (right) showing a region of interval increase in [11C]PBR28 binding corresponding to a gadolinium contrast-enhancing lesion (arrows). d Post-contrast T1weighted MRI (left) and coregistered [11C]PBR28 VT difference map (center) with increased [11C]PBR28 binding that precedes a gadolinium enhancement of the same region a month later (right) (from [15], with permission)

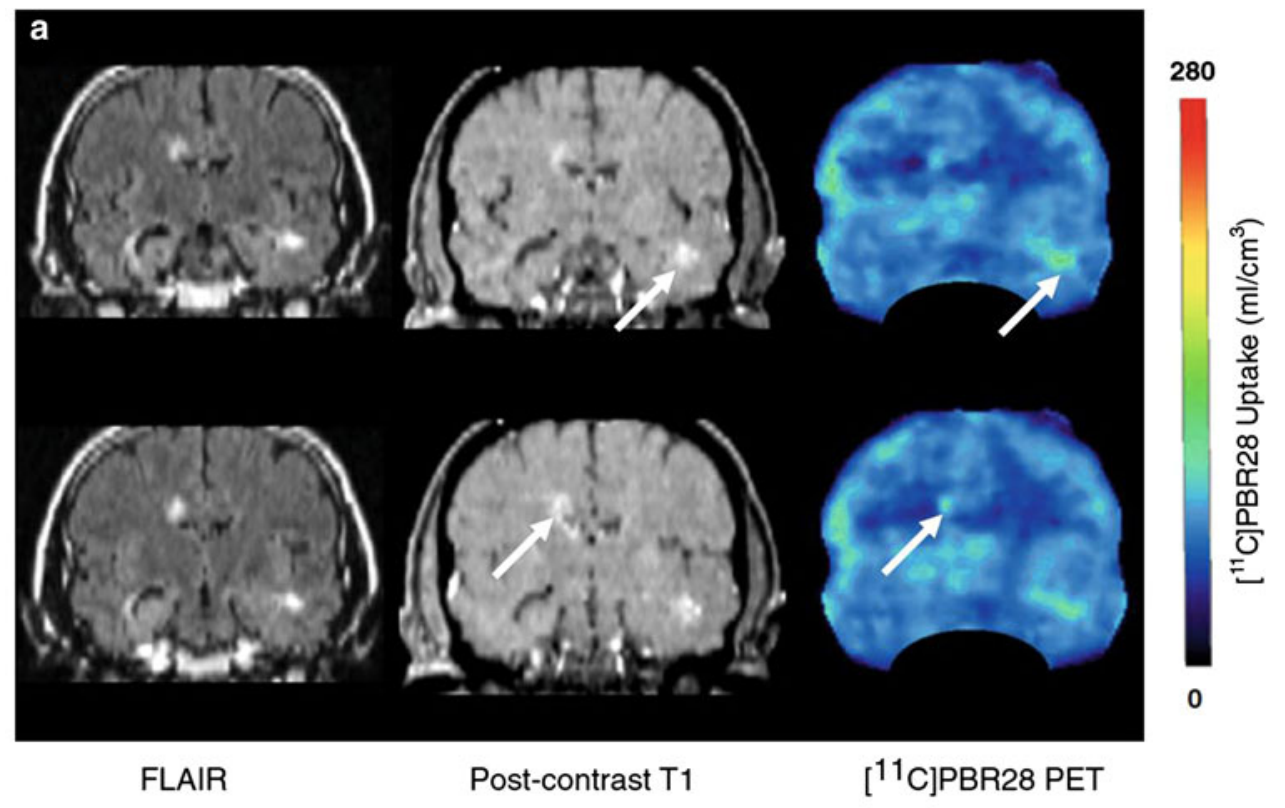

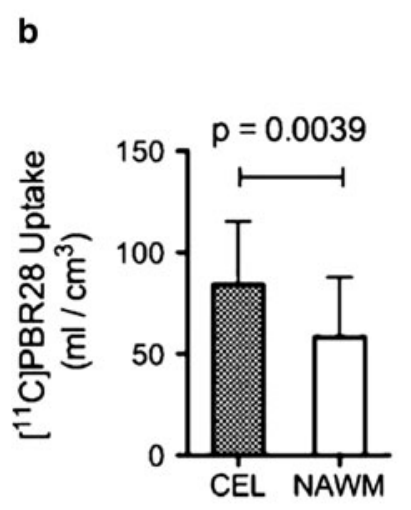

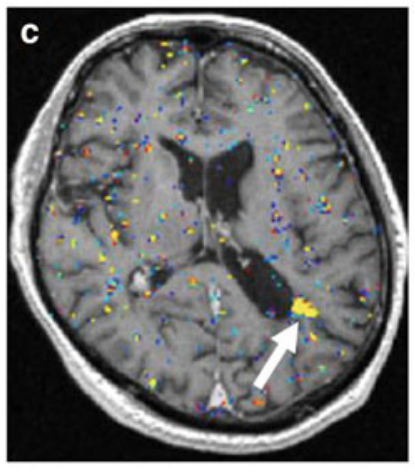

$\left[{ }^{11} \mathrm{C}\right]$ PBR28 $V_{\mathrm{T}} / f_{\mathrm{P}}$ difference map

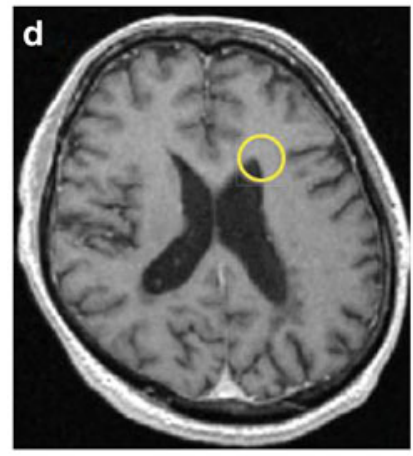

Baseline MRI

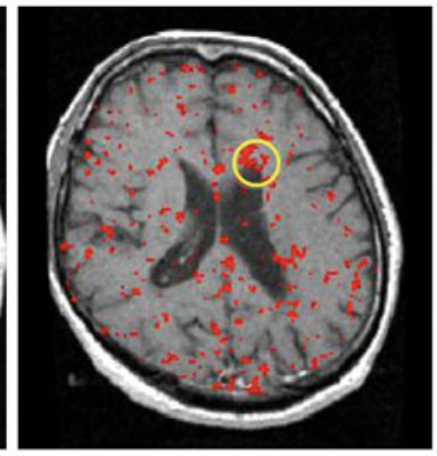

Baseline [ $\left.{ }^{11} \mathrm{C}\right]$ PBR28 PET; gray matter masked

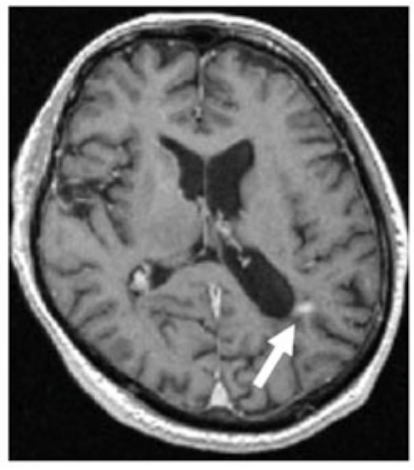

Post-contrast T1

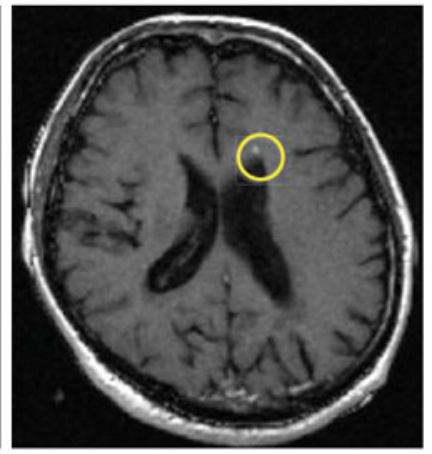

Follow-up MRI beginning to demonstrate a relationship between TSPO genotype and pregnenolone production in anxiety and depression [18]. If these results are confirmed, TSPO imaging could identify early inflammatory changes in depression followed by treatment with anti-inflammatory 
medications. TSPO is being applied to other inflammatory disorders as well, including rheumatoid arthritis [19].

\section{Inflammation in AD}

While brain amyloid continues to represent the main therapeutic target for treatment of $\mathrm{AD}$, pathological and epidemiological studies have also suggested a role for inflammation [20]. These insights led to a series of studies using prednisone and NSAIDs, but there was a failure to reduce cognitive decline in $\mathrm{AD}$. Nonetheless, a focus on inflammatory mechanisms in AD continues, particularly regarding the actions of cytokines and activated microglia [20]. There may be direct activation of brain inflammatory mechanisms and more generalized effects due to immunological dysregulation in aging. Blood cytokines including, IL-1, -6, and $\mathrm{TNF} \alpha$, and C-reactive protein are elevated in some AD patients. It is, however, difficult to separate $\mathrm{AD}$ affects from those due to generalized aging and immune effects. Interestingly though, it has recently been demonstrated that a marker for monocyte/macrophage activation, monocyte chemoattractant protein-1 (MCP-1), is significantly elevated in $\mathrm{AD}$ patients compared to carefully matched elderly controls [21]. Similarly, the receptor for MCP-1, chemokine receptor 2, was down regulated. Thus, the system may offer yet another entry point studying inflammatory mechanisms of AD.

Recently, the role of genes related to inflammatory markers has been evaluated. TREM2 (triggering receptor expressed on myeloid cells 2) is a protein on the surface of myeloid and other cells that interacts with the proteins from the TYROBP gene to transmit chemical signals that mediate phagocytosis during injury or disease. Recently, two studies have demonstrated polymorphisms that are related to late onset $\mathrm{AD}[22 \bullet \bullet, 23 \bullet \cdot$. For comparison, the $\varepsilon 4$ allele of apolipoprotein $\mathrm{E}$ is the best-known genetic variation related to late onset $\mathrm{AD}$ [24]. The two groups identified the TREM2 variant R47H that was closely coupled to an increased risk for late onset $\mathrm{AD}$. In the TgCRND8 mouse model, TREM2 was found to be localized to microglia around plaques and neurons. Significantly, individuals not having $\mathrm{AD}$ who were carriers for the TREM2 variant had an approximately $50 \%$ greater cognitive decline with aging compared to subjects without the variant (Fig. 2) [23••]. The genetic variant may reduce the antiinflammatory effects mediated by TREM 2 and thereby impair the containment of brain inflammatory processes.

While the inflammatory mechanisms involved in the TREM2 variant are different than those with TPSO binding, the common thread of inflammation suggests a potential role of TSPO imaging in AD. Whereas an early study using ${ }^{11} \mathrm{C}$ PK11195 demonstrated elevated cortical binding consistent with microglial activation and AD [25], a recent study with a third-generation TSPO tracer, ${ }^{18}$ F-FEDAA1106, did not show a significant increase [26]. Possible explanations include differences in the two tracers and the clinical populations, but additional investigation will be required to resolve the role of TSPO mechanisms in AD. A study in a rodent model of related neurodegenerative disorder, Parkinson's disease, suggests a potential role of TSPO imaging, but clinical studies have not yet been conducted [27].

\section{Conclusion}

Amyloid and inflammation imaging are robust areas of investigation that are likely to lead to new advances in the diagnosis and therapy of prevalent and inadequately treatable diseases, including AD, multiple sclerosis, Parkinson's disease and depression. Commercial development of new amyloid and TSPO imaging probes will greatly advance this goal and improve patient care. A key metric to follow will be the extent to which early anti-amyloid treatment in MCI patients with amyloid positive scans forestalls or prevents the development of AD. If this approach is also ineffective, new theories and treatment approaches for $\mathrm{AD}$ will be required, possibly based on brain inflammatory mechanisms. Current efforts in multiple sclerosis and other inflammatory brain disorders should provide a solid platform for improved understanding of inflammatory mechanisms in AD. In addition, new molecular imaging probes based on the results of ongoing genetic investigations will also be needed, possibly for TREM2 or related recognition sites. Parallel progress in targeted molecular imaging and new drug development should advance mechanistic understanding and individualized treatment.

Acknowledgments Ms. Nancy Chambers for manuscript editing.

Disclosure J. James Frost declares that he has no conflict of interest.

Human and Animal Rights and Informed Consent This article does not contain any studies with human or animal subjects performed by the author.

\section{References}

Papers of particular interest, published recently, have been highlighted as:

- Of importance,

- Of major importance.

1. Buckley C, Ikonomovic M, Smith A, et al. Flutemetamol F 18 injection PET images reflect brain beta-amyloid levels [abstract]. Alzheimers Dement. 2012;8(Suppl):90. 
2. Clark CM, Pontecorvo MJ, Beach TG, et al. Cerebral PET with florbetapir compared with neuropathology at autopsy for detection of neuritic amyloid-beta plaques: a prospective cohort study. Lancet Neurol. 2012;11(8):669-78.

3. Clark CM, Schneider JA, Bedell BJ, et al. Use of florbetapir-PET for imaging beta-amyloid pathology. JAMA. 2011;305(3):275-83.

4. - Rowe CC, Pejoska S, Mulligan RS, et al. Head-to-head comparison of ${ }^{11} \mathrm{C}-\mathrm{PiB}$ and ${ }^{18} \mathrm{~F}-\mathrm{AZD} 4694$ (NAV4694) for betaamyloid imaging in aging and dementia. J Nucl Med. 2013; 54(6):880-6. This article does a good job demonstrating the current approach to new amyloid probe development and headto-head comparison.

5. Sabbagh M, Seibyl J, Akatsu H, et al. Multicentre phase 3 trial on florbetaben for beta-amyloid brain PET in Alzheimer's disease [abstract]. Alzheimers Dement. 2012;8(Suppl):90.

6. Snitz BE, Weissfeld LA, Lopez OL, et al. Cognitive trajectories associated with beta-amyloid deposition in the oldest-old without dementia. Neurology. 2013;80(15):1378-84.

7. - Johnson KA, Minoshima S, Bohnen NI, et al. Appropriate use criteria for amyloid PET: a report of the Amyloid Imaging Task Force, the Society of Nuclear Medicine and Molecular Imaging, and the Alzheimer's Association. Alzheimers Dement. 2013; 9(1):e-1-16. An important article on clinical use criteria for amyloid imaging agents.

8. Mitka M. PET imaging for Alzheimer disease: Are its benefits worth the cost? JAMA. 2013;309(11):1099-100.

9. Rupprecht R, Papadopoulos V, Rammes G, et al. Translocator protein $(18 \mathrm{kDa})(\mathrm{TSPO})$ as a therapeutic target for neurological and psychiatric disorders. Nat Rev Drug Discov. 2010;9(12):971-88.

10. Kreisl WC, Fujita M, Fujimura Y, et al. Comparison of [(11)C](R)-PK 11195 and [(11)C]PBR28, two radioligands for translocator protein $(18 \mathrm{kDa})$ in human and monkey: implications for positron emission tomographic imaging of this inflammation biomarker. Neuroimage. 2010;49(4):2924-32.

11. Wadsworth H, Jones PA, Chau WF, et al. [(1)(8)F]GE-180: a novel fluorine-18 labelled PET tracer for imaging translocator protein $18 \mathrm{kDa}$ (TSPO). Bioorg Med Chem Lett. 2012;22(3): 1308-13.

12. Owen DRJ, Gunn RN, Rabiner EA, et al. Mixed-affinity binding in humans with $18-\mathrm{kDa}$ translocator protein ligands. J Nucl Med. 2011;52(1):24-32.

13. Politis M, Su P, Piccini P. Imaging of microglia in patients with neurodegenerative disorders. Front Pharmacol. 2012;3:96.

14. Takano A, Piehl F, Hillert J, et al. In vivo TSPO imaging in patients with multiple sclerosis: a brain PET study with [18F]FEDAA1106. EJNMMI Res. 2013;3(1):30.
15. Oh U, Fujita M, Ikonomidou VN, et al. Translocator protein PET imaging for glial activation in multiple sclerosis. J Neuroimmune Pharmacol. 2011;6(3):354-61.

16. Hannestad J, Gallezot JD, Schafbauer T, et al. Endotoxin-induced systemic inflammation activates microglia: [(1)(1)C]PBR28 positron emission tomography in nonhuman primates. Neuroimage. 2012;63(1):232-9.

17. Dowlati Y, Herrmann N, Swardfager W, et al. A meta-analysis of cytokines in major depression. Biol Psychiatry. 2010;67(5):446-57.

18. Costa B, Pini S, Abelli M, et al. Role of translocator protein $(18 \mathrm{kDa})$ in adult separation anxiety and attachment style in patients with depression. Curr Mol Med. 2012;12(4):483-7.

19. van der Laken CJ, Elzinga EH, Kropholler MA, et al. Noninvasive imaging of macrophages in rheumatoid synovitis using ${ }^{11} \mathrm{C}$ (R)-PK11195 and positron emission tomography. Arthritis Rheum. 2008;58(11):3350-5.

20. Lane RF, Shineman DW, Steele JW, et al. Beyond amyloid: the future of therapeutics for Alzheimer's disease. Adv Pharmacol. 2012;64:213-71.

21. Zhang R, Miller RG, Madison C, et al. Systemic immune system alterations in early stages of Alzheimer's disease. J Neuroimmunol. 2013;256(1-2):38-42.

22. • Guerreiro R, Wojtas A, Bras J, et al. TREM2 variants in Alzheimer's disease. N Engl J Med. 2013;368(2):117-27. This and the companion article by Jonsson et al. demonstrate important new findings on the TREM2 gene, which may be an important inflammatory risk factor for Alzheimer's disease.

23. • Jonsson T, Stefansson H, Steinberg S, et al. Variant of TREM2 associated with the risk of Alzheimer's disease. N Engl J Med. 2013;368(2):107-16. This and the companion article by Guerreiro et al. demonstrate important new findings on the TREM2 gene, which may be an important inflammatory risk factor for Alzheimer's disease.

24. Skaper SD. Alzheimer's disease and amyloid: culprit or coincidence? Int Rev Neurobiol. 2012;102:277-316.

25. Cagnin A, Brooks DJ, Kennedy AM, et al. In-vivo measurement of activated microglia in dementia. Lancet. 2001;358(9280):461-7.

26. Varrone A, Mattsson $\mathrm{P}$, Forsberg A, et al. In vivo imaging of the $18-\mathrm{kDa}$ translocator protein (TSPO) with [(18)F]FEDAA1106 and PET does not show increased binding in Alzheimer's disease patients. Eur J Nucl Med Mol Imaging. 2013;40(6):921-31.

27. Maia S, Arlicot N, Vierron E, et al. Longitudinal and parallel monitoring of neuroinflammation and neurodegeneration in a 6-hydroxydopamine rat model of Parkinson's disease. Synapse. 2012;66(7):573-83. 